[3] Buruiana L. M. et V. Pavlu. Naturwiss. 1957, 22, 589.

[4] Buruiana L. M. et V. Pavlu. Naturwiss. 1958, 6, 132.

[5] Buruiana L. M. et V. Pavlu. Studii si Cercetari de Chimie, Acad. R.P.R. 1958, Anul VI. No 1, 139.

[6] Chris ensen L. R. Arch. Biochim. Biophys. 1954, 53, 128.

[7] Danilewsky A. I. et Radenhausen. Forschung. a. d. Gebiete d. Viehhalt. $1885,2,1$.

[8] Frazer D et R. E. Powell. J. of Biol. Cjem. 1950, 187, 203.

[9] Hipp. N. I., M. L. Groves, I. H. Custer et T. L. Me. Meekin. J. of Dairy Sci. 1952, 35, n० 3, 272.

[10] Linderström-Lang K. et S. Kodama. Compt. rend. trav. Lab. Carlsberg Ser. Chim. 1925, 1, 16.

[11] Mellander. O. Biochem. $Z$ 1939, 300, 240.

[12] Northrop J. H. J. Gen. Physiol. 1933, 16, 339.

[13] Striks W. et I. M. Kolthoff. Sbornik Mezinaroniho. Polarographicenho Sjezdu v Praze. 1st Congress. 1951, 386.

\title{
DE LA DESTRUCTION DES BACTÉRIES PAR LA CHALEUR ETUDE DE L'EFFICACITÉ DE LA PASTEURISATION DU LAIT
}

par

A. NEVOT, Ph. LAFONT et J. LAFONT

Comme suite à la présentation de notre monographie sur la thermo-résistance des germes pathogènes dans le lait (séance de l'Académie du 27 janvier) (1), j'ai l'honneur de vous exposer nos protocoles expérimentaux et les résultats obtenus.

Notre travail comporte d'abord une étude faite, au laboratoire, sur la sensibilité à la chaleur des bactéries pathogènes ou commensales pouvant contaminer le lait, puis une étude effectuée en utilisant, pour le traitement thermique de laits artificiellement pollués, une installation expérimentale de pasteurisation comparable, quant à son principe de fonctionnement, aux appareils industriels de type H.T.S.T. La pasteurisation H.T.S.T. (High Temperature Short Time), la plus répandue dans les pays évolués, consiste en un chauffage du lait, en couche mince et en courant continu, à $71^{\circ}-72^{\circ}$, pendant au moins quinze secondes.

\section{I. - Etude de la sensibilité à la chaleur des bactéries pathogènes ou commensales pouvant contaminer le lait}

$\mathrm{Au}$ laboratoire nous avons adopté le protocole expérimental suivant :

C.R. Acad, Méd, 1959, 143, 175.

(1) Le Lait, 1959, 39, 170 
a). Un bain-marie à eau, réglé à la température désirée, est garni de tubes en verre d'un diamètre intérieur de 11 millimètres; chaque tube contient $1 \mathrm{ml} .9$ d'eau physiologique stérile, de lait stérilisé par autoclave ou de lait cru. Ces tubes sont placés dans le bain-marie pendant un temps suffisamment long pour qu'au moment de la manipulation proprement dite, l'équilibre thermique entre leur contenu et l'eau de l'appareil soit atteint. L'obtention de cet équilibre est vérifié par l'emploi d'un couple thermo-électrique.

b) Au temps 0 , un volume de $0 \mathrm{ml} .1$ de la suspension du germe étudié est injecté à la pipette dans la masse de liquide préchauffé (eau physiologique ou lait), contenu dans chacun des tubes.

c) Après un laps de temps arbitraitement déterminé, les germes sont soustraits à l'action de la chaleur par adjonction rapide, dans le tube, de 2 millilitres d'eau physiologique froide. Sans marquer aucun temps d'arrêt, 2 millilitres du mélange ainsi obtenu sont aspirés dans une pipette froide et servent à effectuer la recherche des germes survivant au chauffage.

Des courbes de variations de la température, d'une part, lors de l'introduction de $0 \mathrm{ml}$. 1 d'eau physiologique à $15^{\circ}$ dans un volume dix-neuf fois supérieur de liquide préalablement chauffé, d'autre part, lors de l'introduction de 2 millilitres d'eau physiologique à $15^{\circ} \mathrm{C}$ dans un tube contenant 2 millilitres de liquide (eau ou lait), placé au bain-marie à une température comprise entre $56^{\circ} \mathrm{C}$ et $80^{\circ} \mathrm{C}$ figurent dans la monographie.

Les suspensions bactériennes soumises au chauffage ont été dans la plupart de nos expériences réalisées à partir de cultures de vingt-quatre heures à $37^{\circ} \mathrm{C}$ en bouillon nutritif.

Après introduction de $0 \mathrm{ml} .1$ dans $1 \mathrm{ml} .9$ de liquide préchauffé, la suspénsion obtenue titrait régulièrement plus de $1,10^{7}$ germes par millilitre. En ce qui concerne le B. K., les chauffages ont été effectués sur des suspensions réalisées à partir de cultures, de dix jours environ, en milieu liquide de Dubos au Twen 80, cultures titrant environ I milligramme de bacilles par millilitre; pour chaque essai, $0 \mathrm{ml}$. 1 a été incorporé à $1 \mathrm{ml}$. 9 d'eau physiologique, de lait stérilisé ou de lait cru préchauffé. Si l'on admet que 1 milligramme de bacilles contient en moyenne $5.10^{7}$ germes, il s'en suit que dans 1 millilitre du liquide de mélange il y a $2,5.10^{5}$, soit 250.000 bacilles tuberculeux

La contamination des laits a été également effectuée avec des produits pathologiques tuberculeux : expectorations humaines, broyats de lésions ganglionnaires de bovins. Dans ce cas la contamination du lait était de l'ordre de 10 à 30 bacilles au millilitre à en juger par le nombre de colonies développées sur milieu de Löwens. tein-Jensen. 
La recherche des bactéries survivantes après chauffage, a été effectuée sur au minimum la moitié, soit 2 millilitres de la suspension Pour chaque germe ont été utilisés des milieux sélectifs appropriés et pour le B. K. particulièrement, à l'ensemencement sur milieu de Löwenstein-Jensen á été associée l'inoculation expérimentale au cobaye.

Pour cet exposé, en ce qui concerne les résultats obtenus, nous ne mentionnons dans le tableau ci-dessous (tableau I) que ceux relatifs aux principaux germes pathogènes : bacilles tuberculeux, Brucelles, Salmonelles. Pour les autres bactéries tous les détails sont contenus dans la monographie.

\section{TABLEAU I}

SENSIBILITÉ A LA GHALEUR DES BAGTÉRIES PATHOGĖNES OU COMMENSALES POUVANT CONTAMINER LE LAIT. TEMPS DE DESTRUCTION A $7^{\circ}$

\begin{tabular}{|c|c|c|}
\hline & En lait cru & En lait stérilisé \\
\hline $\begin{array}{l}\text { en eultures } \ldots \ldots \ldots \ldots \ldots \ldots \ldots \\
\text { en produits pathologiques } \ldots \ldots \ldots \ldots \ldots\end{array}$ & $\begin{array}{c}8^{\prime \prime} \text { à } 12, " \\
16, "\end{array}$ & 9 "' à 12 "' \\
\hline rculeux bovins: & & \\
\hline $\begin{array}{l}\text { en cultures } \ldots \ldots \ldots \ldots \ldots \ldots \ldots \ldots \\
\text { en produits pathologiques } \ldots \ldots \ldots \ldots \ldots\end{array}$ & $\begin{array}{c}4 \text { "' à 8", } \\
12 \text { ", }\end{array}$ & 4"' à 9 "' \\
\hline ucella melitensis $\ldots \ldots \ldots \ldots \ldots \ldots \ldots$ & & 18 ' à $20 "$ \\
\hline acella abortus bovis $\ldots \ldots \ldots \ldots \ldots \ldots$ & & 12 " à 18 "' \\
\hline $\begin{array}{l}\text { le d'Eberth } \ldots \ldots \ldots \ldots \ldots \ldots \ldots \ldots \ldots \ldots \ldots \\
\text { aratyphique B } \ldots \ldots \ldots \ldots \ldots \ldots \ldots\end{array}$ & $\begin{array}{c}\text { 4"' à 5"' } \\
4 \text { ", }\end{array}$ & \\
\hline phylocoque $\ldots \ldots \ldots \ldots \ldots \ldots \ldots$ & 10 " à $11 "$, & 14 "' à 15 ", \\
\hline ptocoque (groupe A) ...... & & 7, \\
\hline rocoqu & 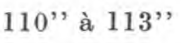 & 110 "' à $113^{\prime}$ \\
\hline
\end{tabular}

\section{II. - Etude de la destruction des bactéries pathogènes ou commensales dans les conditions de chauffage voisines de celles réalisables dans l'industrie lai tière}

Nous avons monté pour cette étude un pasteurisateur de modèle réduit comprenant :

Un échangeur thermique alimenté en eau chaude, assurant l'élévation de la température du lait;

Une série de récipients hermétiquement clos jouant le rôle de chambreurs ;

Un second échangeur thermique parcouru par un courant d'ea $u$ froide destiné à abaisser la température du lait ;

Un système d'alimentation en lait permettant d'assurer un débit constant sous une certaine pression. 
Sur la figure 1 est représenté l'ensemble de cette installation.

Chacun de ces éléments est constitué par un tube cylindrique en aluminium de 5 millimètres de diamètre, calorifugé extérieurement, et dans lequel circule le liquide destiné soit à réchauffer, soit

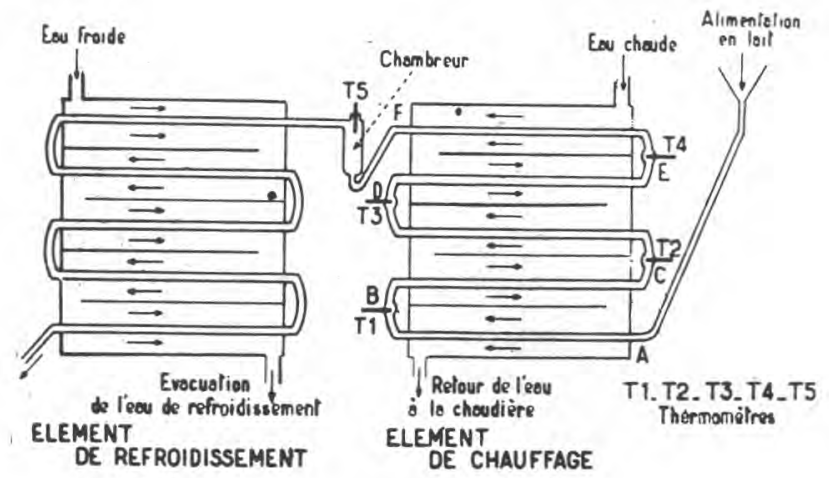

Fig 1

à refroidir le lait. En certains points (B.C.D.E.), la canalisation intérieure se dégage de son enveloppe chauffante et présente un léger renflement destiné à loger le bulbe d'un thermomètre de précision.

Les ampoules utilisées comme chambreurs ont des sections et des volumes différents calculés de telle façon que le lait les traverse respectivement en deux, cinq, dix, vingt et trente secondes.

Tous les essais ont été conduits en réglant la position du système d'alimentation de façon à obtenir un débit, à la sortie de l'élément de refroidissement, de 1.000 millilitres en cent secondes. Cette condition étant fixée, le calcul de la vitesse de circulation du lait dans l'installation a fourni les valeurs numériques suivantes:

Temps de franchissement des distances égales $\mathrm{AB}, \mathrm{BC}, \mathrm{CD}, \mathrm{DE}$, EF : 3,5 secondes.

Temps de franchissement de l'élément chauffant : 17,5 secondes.

Temps de franchissement de l'élément de refroidissement (de même taille que l'élément de chauffage) : 17,5 secondes.

Pour obtenir cette vitesse de passage du lait dans la canalisation de l'appareil, la dénivellation à respecter entre le niveau du lait dans l'entonnoir d'alimentation et le tube supérieur de chaque échangeur thermique est de l'ordre de $1 \mathrm{~m}$. 20. Cette dénivellation est suffisante pour empêcher tout phénomène de moussage du lait dans l'appareil.

Sur le graphique 1 figurent les températures relevées en T1, T2, T3, T4, au cours de chambrage à $80^{\circ}, 75^{\circ}, 72^{\circ}, 68^{\circ}$.

L'appareil expérimental que nous avons utilisé est très compa- 
rable à celui d'une installation industrielle de pasteurisation. Il en diffère par les points suivants :

Le diamètre de la canalisation conduisant le lait est supérieur d'au moins 1 millimètre à l'épaisseur de la couche de lait réalisée dans les appareils industriels à plaques.

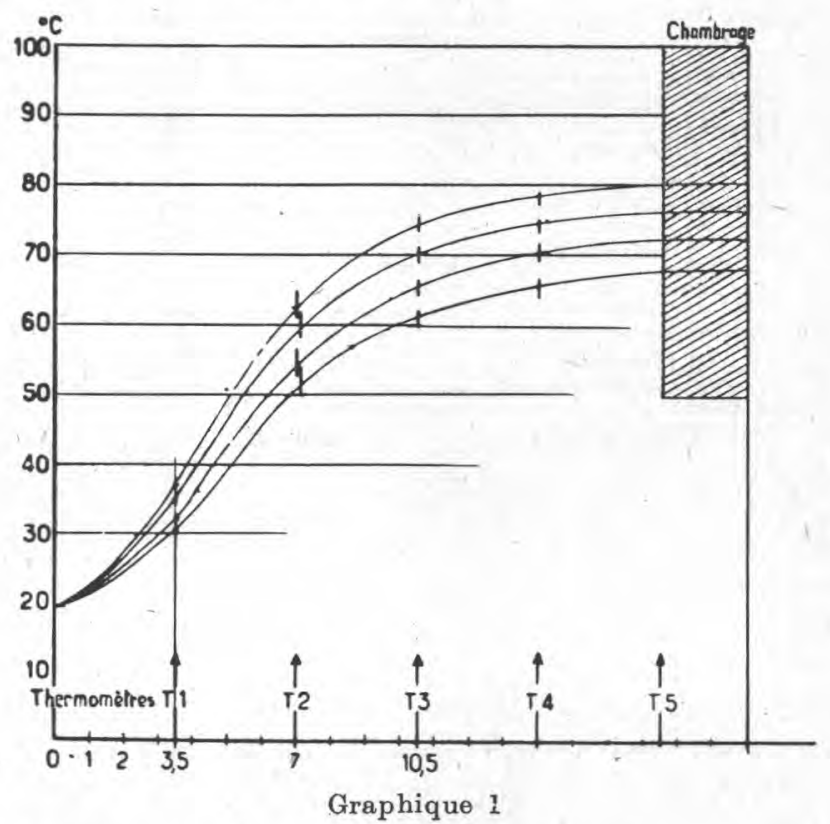

Le refroidissement est beaucoup plus brutal.

Ces deux facteurs peuvent avoir eu une influence sur la valeur des résultats que nous avons obtenus en matière de destruction des bactéries contenues dans le lait, mais il est évident que cette influence ne peut se traduire que par un effet moindre du traitement thermique réalisé dans l'installation expérimentale par rapport à ce qui peut être obtenu avec un appareil industriel.

Dans nos essais le volume du lait employé a été de l'ordre de 40 litres et la richesse des contaminations a été voisine de celle signalée pour les travaux effectués au laboratoire.

Les résultats obtenus dans cette deuxième partie de nos études expérimentales figurent dans le tableau ci-dessous (tableau II) sur lequel ont été reportées les données du tableau I, aux fins de comparaison.

La grande différence entre le temps de destruction des germes dans nos essais de laboratoire et dans nos essais industriels est évidente. La raison en est fort simple. Au laboratoire, les laits contaminés sont portés d'emblée à la température voulue; $72^{\circ}$, par 
exemple, pendant un temps bien déterminé, puis refroidis très rapidement. La courbe de chauffage est ainsi de type rectangulaire : ascension brusque de la température du lait traité de $20^{\circ}$ à $72^{\circ}$,

\section{TABLEAU II}

SENSIBILITÉ A LA GHALEUR DES BAGTÉRIES PATHOGÈNES OU COMMENSALES POUVANT GONTAMINER LE LAIT.

TEMPS DE DESTRUCTION A $72^{\circ}$

\begin{tabular}{cc}
\multicolumn{2}{c}{ En lait cru } \\
Appareil & expérimentation \\
de pasteurisation & de laboratoire
\end{tabular}

Bacilles tuberculeux humains :

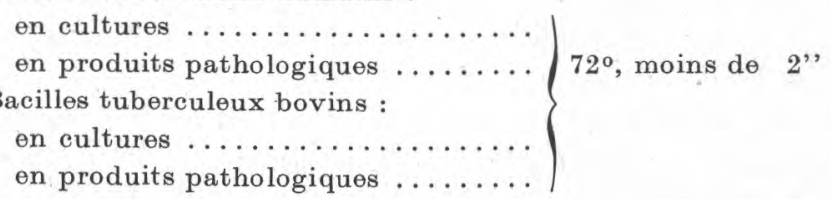

$$
\begin{gathered}
8 \text { " à } 12 \text { "' } \\
16 \text { "' } \\
\text { 4" à 8"' } \\
12 \text { ", }
\end{gathered}
$$

Brucella melitensis

$72^{\circ}$, moins de $5^{\prime \prime}$

$$
\begin{gathered}
18 \text { "' à } 20^{\prime \prime} \\
12 \text { " à } 18 \text { ", } \\
4 \text { "' à } 5 \text { ", } \\
4 \text { "' } \\
10 \text { "' à } 11 \text { ", } \\
7 \text { ", }
\end{gathered}
$$

Streptocoque (groupe A) $\ldots \ldots \ldots \ldots$

Entérocoque $\ldots \ldots \ldots \ldots \ldots \ldots \ldots \ldots \ldots \ldots 2^{\circ}$, moins de $60^{\prime}$,

$110^{\prime \prime}$ à $113^{\prime \prime}$

maintien à cette température pendant le temps choisi : 2", 5", 10", par exemple, puis refroidissement brusque. Dans notre traitement de type industriel, le lait avant d'atteindre la température voulue

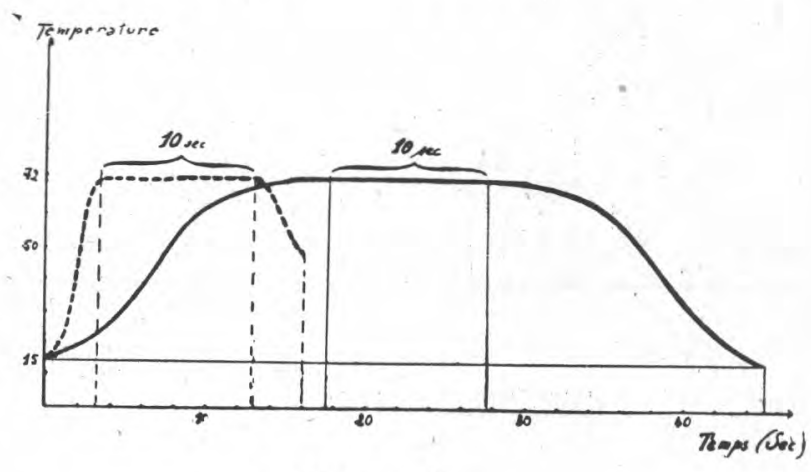

\section{Gapmiour 2}

subit un préchauffage de $20^{\circ}$ à $72^{\circ}$ pendant exactement $17,5^{\prime \prime}$, il est maintenu à cette dernière température pendant le temps fixé expérimentalement. De même le refroidissement jusqu'à environ 
$20^{\circ}$ se produit également en $17,5^{\prime}$, si bien que le liquide en plus de la pasteurisation proprement dite $\left(72^{\circ}\right.$ pendant $10^{\prime}$ par exemple), est porté durant $35^{\prime \prime}$ entre $20^{\circ}$ et $72^{\circ}$, plus précisément pendant $21^{\prime \prime}$ entre $50^{\circ}$ et $72^{\circ}$, ce qui permet de se rendre compte des grandes différences entre les résultats du laboratoire et ceux de l'industrie. La courbe de ce chauffage dans ce dernier cas est de type trapézoïdal. Le graphique 2 illustre nos explications.

Ces faits exposés, existe-t-il un moyen permettant de reconnaître si un lait a été effectivement pasteurisé, c'est-à-dire débarrassé de toute flore nocive. Enregistrer la température obtenue et son temps d'action est un procédé, mais il existe une méthode plus simple et plus sûre qui permet ce contrôle : c'est la recherche de la phosphatase alcaline dans les laits pasteurisés.

La phosphatase est un enzyme toujours présent dans le lait cru, enzyme qui est inactivé par un chauffage supérieur à celui nécessaire pour la destruction des germes pathogènes, notamment du bacille tuberculeux.

Le graphique 3 montre que pour inhiber la phosphatase, il faut pratiquer un chauffage plus élevé que celui nécessaire pour

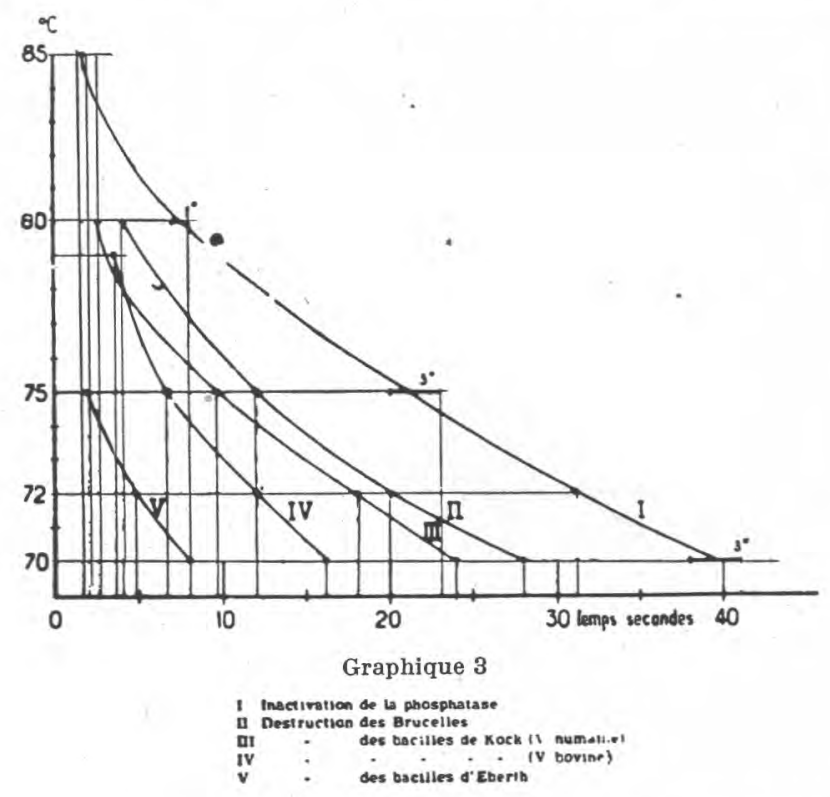

détruire les brucelles, les bacilles tuberculeux humain et bovin, les bacilles typhique et paratyphiques.

L'absence de phosphatase alcaline dans un lait pasteurisé est donc une garantie de la destruction des germes pathogènes. Et au 
lieu de fixer des températures et des temps de, chauffage pour définir des laits correctement pasteurisés, il serait plus logique de déclarer que tout lait effectivement pasteurisé est exempt de phosphatase alcaline.

Notre conclusion est la suivante :

$1^{\circ}$ Un lait correctement pasteurisé est exempt de germes pathogènes.

$2^{\circ}$ Le problème de la pasteurisation du lait est résolu du point de vue industriel.

$3^{\circ}$ L'épreuve de la phosphatase, lorsqu'elle est négative, est une garantie de pasteurisation efficace.

Aussi comprend-on notre étonnement en face de l'opinion d'hygiénistes français, non spécialistes des questions laitières, qui soutiennent qu'un lait même correctement pasteurisé doit, avant sa consommation, être soumis à une ébullition de cinq minutes, afin de le débarrasser des germes pathogènes qu'il peut éventuellement contenir. Cette opinion est en contradiction avec tous les travaux scientifiques effectués sur ce problème tant à l'étranger qu'en France.

Si l'on recommande l'ébullition du lait pasteurisé, il ne faut pas que cette mesure soit motivée par une incertitude quant à la destruction des germes pathogènes, mais par le fait qu'un lait qui, à la sortie du pasteurisateur offre toutes garanties, peut être souillé secondairement lors de son conditionnement.

On sait que la stérilisation des bouteilles destinées à recevoir le lait pasteurisé ne peut être réalisée d'une manière absolue, mais actuellement dans beaucoup de pays l'emploi du "récipient perdu " en carton paraffiné ou en carton doublé intérieurement d'une mince feuille d'aluminium ou d'une pellicule thermoplastique de polyéthylène pur apparaît comme un réel progrès. Ces récipients peuvent être préparés à l'avance, mais peuvent aussi être mis en forme immédiatement avant le remplissage ou même pendant ce remplissage, ce qui diminue considérablement les possibilités de souillures, en germes pathogènes notamment. 